HOT GAS IN THE LISM: OPTICAL, UV, AND EUV WAVELENGTHS 


\title{
OBSERVATIONS OF ABSORPTION LINES FROM HIGHLY IONIZED ATOMS
}

\author{
Edward B. Jenkins \\ Princeton University Observatory \\ Princeton, N. J. 08544
}

\begin{abstract}
In the ultraviolet spectra of hot stars, we can see absorption lines from highly ionized species in the interstellar medium. Observations of these features have been very influential in revising our perception of the medium's various physical states and how gases in space are heated and ionized. The pervasiveness of $O$ VI absorption lines, coupled with complementary observations of a diffuse background in soft $x$-rays and EUV radiation, shows that there is an extensive network of low density gas $\left(n \approx\right.$ few $\left.\times 10^{-3} \mathrm{~cm}^{-3}\right)$ existing at "coronal" temperatures, $5.3 \leqslant \log \mathrm{T} \leqslant 6.3$. Thus, while we once thought that $x$-rays and cosmic rays were the dominant sources of excitation and heating of the interstellar gas, we now realize that shocks created by supernova explosions or mass loss from early-type stars can propagate freely through space and eventually transfer a large amount of energy to the medium. To create the coronal temperatures, the shocks must have velocities in excess of $150 \mathrm{~km} \mathrm{~s}^{-1}$; shocks at somewhat lower velocity ( $\left.\mathrm{s} 100 \mathrm{~km} \mathrm{~s}^{-1}\right)$ can be directly observed in the lines of Si III. Observations of other lines in the ultraviolet, such as Si IV and C IV, may highlight the widespread presence of energetic uv radiation from very hot, dwarf stars. More advanced techniques in visible and $x$-ray astronomical spectroscopy may open up for inspection selected lines from atoms in much higher stages of ionization.
\end{abstract}

\section{BEGINNINGS}

Well before space-borne instruments were launched to observe ultraviolet absorption lines from the interstellar medium, it was suggested that features from highly ionized atoms might provide a valuable insight on the magnitude and character of ionizing and heating processes which were responsible for the observed physical states of gases in space. Early proposals centered on the notion that primarily neutral gas (HI regions) occupied most of the space, and that low energy cosmic ray particles or x-rays were responsible for the observed temperature and small traces of ionization (Pikel'ner 1967, Spitzer 1968, Spitzer and Tomasko 1968, Field, et al. 1969, Spitzer and Scott 1969, Goldsmith, et al. 1969, Silk and Werner 1969, Dalgarno and McCray 1972). Since low energy cosmic rays are shielded from us by interplanetary magnetic flelds and low energy x-rays were difficult to measure with existing experiments, we could not measure the strength of these proposed sources of heating and partial ionization. Instead, it was suggested that the fluxes could be ascertained indirectly by observing the interstellar abundances of some highly ionized forms of particular heavy elements, such as C III, C IV, N III, N 
V, Si III, Si IV, S III and S IV, all which had strong resonance lines in the ultraviolet (Silk 1970, Silk and Brown 1971, Weisheit 1973).

A few years after a search for such absorption features in stellar spectra had been initiated by the Copernicus satellite, Steigman (1975) cautioned that the highly charged ions could be severely depleted in HI regions if, as seemed likely, the cross section for charge exchange with the neutral atoms were reasonably high. This effect could be much more important than recombinations with free electrons considered earlier. In the years which followed, Steigman's warning was shown to be valid: Blint, et al. (1976) calculated $\langle\sigma v\rangle \approx 10^{-6} \mathrm{~cm}^{3} \mathrm{~s}^{-1}$ for charge exchanges between $C I V$ and $H$ for temperatures between 1000 and $20,000 \mathrm{~K}$, and similar conclusions for $\mathrm{N} \mathrm{IV}$, the primary reservoir for the observable $\mathrm{N} \mathrm{V}$, were derived by Christensen, et al. (1977). These calculations using the scattering approximation were valid only for impact energies $\sim 1 \mathrm{eV}$, but Watson, et al. (1979) carried out quantal calculations which showed that the large cross sections persisted even to energies which were characteristic of temperatures in ordinary, dense interstellar clouds $(T \sim 100 \mathrm{~K})$.

While these theoretical conclusions muted one particular incentive for observing highly ionized atoms in the interstellar medium, ${ }^{(1)}$ the Copernicus researchers were also interested in the prospect of detecting low density, thermally ionized gases which could conceivably inhabit some voids between the clouds of ordinary, cool H I. Their efforts were rewarded by an early indication of absorption near the wavelength of the $1032 \AA$ feature of $O \mathrm{VI}$ in the spectra of $\alpha$ Eri and $\lambda$ Sco, reported by Rogerson, et al. (1973). There soon followed definitive studies which showed that both members of the 0 VI doublet could be seen in many stars, that the absorptions were very likely interstellar, and that the high state of ionization was created by collisions in a hot plasma, instead of cosmic ray or x-ray bombardment in an ordinary cool gas (York 1974, Jenkins and Meloy 1974). From the ubiquity of the 0 VI lines and their large apparent widths, coupled with a lack of conspicuous features from other ions having a slightly lower ionization potential, these authors concluded that low density gases having a temperature somewhere in the interval $2 \times 10^{5}$ to $2 \times 10^{6} \mathrm{~K}$ were pervasive.

The $O$ VI findings were published concurrently with an article by Williamson, et al. (1974), who suggested that the diffuse, soft $x$-ray background radiation observed from their sounding-rocket experiments originated from the same very hot medium, although their measurements were mostly sensitive to emissions from gases at the upper end of the temperature range, 1 to several $\times 10^{6} \mathrm{~K}$. These two fundamentally different experimental results, the detections of uv absorption features and the emissions at x-ray energies, were important milestones which precipitated a new era of thought on the state of the interstellar medium. In many respects, the widespread existence of hot material was reminiscent of a proposal by Spitzer (1956) that the corona of our galaxy is comprised of a very low density medium in a temperature regime similar to that of the solar corona. ${ }^{(\mathcal{})}$

(1) Other ways were found to measure indirectly the cosmic ray and x-ray ionization rates in the interstellar medium, using the abundances of $\mathrm{HD}$ and $\mathrm{OH}$ which are influenced by the proton densities (O'Donnell and Watson 1974, Jura 1974, Black and Dalgarno 1973).

(2) Unfortunately, the Copernicus satellite lacked the sensitivity to measure the spectra of sources more than 1 or $2 \mathrm{kpc}$. from the galactic plane, so the structure of a corona of our galaxy could not be investigated. Recent data from the IUE satellite show evidence for an excess of Si IV and C IV in high latitude sources, indicating the presence of material at "transition layer" type temperatures (Savage and deBoer 1979, 1981, Pettini and West 1982), but the wavelength cover- 


\title{
II. INVESTIGATION OF THE O VI-BEARING GAS
}

\author{
a) Origins: Stellar, Circumstellar or Interstellar?
}

The first, and perhaps most critical challenge for those who advocate an interstellar interpretation for certain absorption lines is to convince the skeptics that the stars themselves, or their influences on the environment, are not responsible for the observed features. This problem is not a new one; controversy in this area extends back to the turn of the century when the first interstellar lines, those of Ca II, were identified in the spectra of binary stars (Hartmann 1904, Slipher 1909). For the $O$ VI features, which must arise from material which is subjected to strong heating, a resolution of circumstellar versus interstellar origins is particularly relevant, inasmuch as the $O$ and $B$ type stars which produce enough radiation to show features at 1032 and $1038 \AA$ are the very stars which must deliver a large amount of energy to the environment. Plausible theoretical interpretations of the observed O VI column densities were advanced for both viewpoints: The O VI could reside in zones associated with the target stars or whole groups of stars in the association (the "interstellar bubbles" of Castor, et al, 1975; also see Weaver, et al. 1977) or, alternatively, there could be an interconnected network of regions in space heated, on successive occasions, by supernova blast waves (the "interstellar tunnels" of Cox and Smith 1974).

While the atmospheres of hot stars are responsible for broad $0 \mathrm{VI}$ absorption features, often with P-Cygni type characteristics, the radial velocities of the narrower features identified as "interstellar" show no correlation with stellar velocities (Jenkins and Meloy 1974, Jenkins 1978b), nor are the O VI lines influenced in any way by the stars' projected rotational velocities, $v \sin i$. In addition, York (1977) studied the $O$ VI feature in the spectrum of the spectroscopic binary $\lambda$ Sco and found no variation with orbital phase.

The possibility that $\mathrm{OVI}$ is circumstellar is a more difficult issue to address. A detailed discussion of the observational clues was presented by Jenkins (1978c), based on a comprehensive survey of O VI lines (Jenkins 1978a) which followed the earlier discoveries. Briefly, Jenkins concluded that most of the 0 VI came from truly interspersed material, but that some of the absorptions might be attributable to the evaporation zones within the very hot cavities created by the high velocity mass loss from the stars being observed, as described by Castor, et al. (1975) and Weaver, et al. (1977).

An interesting outgrowth of Jenkins's (1978c) analysis which is relevant to the local interstellar medium is that the general rate of increase of the $\mathrm{O} V \mathrm{VI}$ column density $N(\mathrm{O} \mathrm{VI})$ with distance $r$, while indicating an average density $n(0 \mathrm{VI})=$ $1.7 \times 10^{-8} \mathrm{~cm}^{-3}$ but with considerable scatter, favors the existence of a positive intercept with $N(O$ VI $)$ of around $5 \times 10^{12} \mathrm{~cm}^{-2}$ at $r=0 .{ }^{(3)}$ This offset could be

\footnotetext{
age of IUE does not extend down to the $O$ VI lines, so the structure of material at "coronal" temperatures has yet to be determined. This paper will not dwell on the conclusions about gas away from the galactic plane, since the theme of this volume is on the properties of the local interstelIar medium.

(3) The few lines of sight with anomalously high column densities (containing Jenkins's [1978b] "second population" regions) were excluded in this study. If one were to include these regions and also insist the intercept is really zero, the average $n\left(O\right.$ VI) increases to $2.8 \times 10^{-8} \mathrm{~cm}^{-3}$.
} 
interpreted either as an excess resulting from the circumstellar bubbles around many of the stars, or as a contribution from a local concentration of hot gas in our neighborhood which has a disproportionate influence because all of the lines of sight, by necessity, emanate from a single point instead of being randomly scattered about.

\section{b) General Properties}

A striking feature of the 0 VI profiles seen in the survey of 72 stars reported by Jenkins (1978a) is that the dispersion of radial velocities is much smaller than that expected from the shock speeds of $v \sim 150 \mathrm{~km} \mathrm{~s}^{-1}$ needed to heat the gas to the temperatures $T \sim 3 \times 10^{5} \mathrm{~K}$ for collisionally ionizing oxygen to +5 . A composite of all the profiles extending over a total distance of about $50 \mathrm{kpc}$. to all of the stars had an $r m s$ spread in radial velocity of $28 \mathrm{~km} \mathrm{~s}^{-1}$, after applying small corrections to compensate for differential galactic rotation. Moreover, Jenkins (1978b) examined the statistical behavior of the velocity centroids and widths of profiles seen in the individual lines of sight, and he concluded that the distributions were consistent with the notion that individual packets of $\mathrm{OVI}$, each with a column density of about $10^{13} \mathrm{~cm}^{-2}$ and internal broadening of $10 \mathrm{~km} \mathrm{~s}^{-1}$, moved about at random with an $r m s$ dispersion of radial velocities of only $26 \mathrm{~km} \mathrm{~s}^{-1}$. Large fluctuations in average $\mathrm{O}$ VI densities to different stars independently supported the idea of discrete domains of $O$ VI-bearing gas; such variations were in accord with the expected Poisson statistical fluctuations.

While appreciable concentrations of $0 \mathrm{VI}$ can exist in collisional equilibrium for temperatures in the range $1.7 \times 10^{5}-2.0 \times 10^{6} \mathrm{~K}$ (Shull and Van Steenberg 1982), the relative lack of accompanying $\mathrm{N} \mathrm{V}$ absorptions (York 1977) indicates that most of the material is at $T>2.5 \times 10^{5} \mathrm{~K}$, since $\mathrm{N} V$ has its peak fractional abundance at $T \sim$ $1.8 \times 10^{5} \mathrm{~K}$. The narrowness of many of the individual profiles $\left(\left\langle v^{2}\right\rangle^{k} \leqslant 14 \mathrm{~km} \mathrm{~s}^{-1}\right)$, however, puts a restriction on the amount of thermal doppler broadening present, which means $T<16 m_{p}\left\langle v^{2}\right\rangle / k=3.8 \times 10^{5} \mathrm{~K}$. Actually, the proposition that the hot gas is nearly isothermal is probably overly simplistic and has no compelling theoretical justification. (4) Jenkins (1978b) showed that the effects from the above mentioned observational constraints, when integrated over the peak of the curve for the $\mathrm{O}^{+5}$ ionization fraction with temperature, still permitted a power-law distribution in temperature for the electron density $n_{e}$, with $d n_{\varepsilon} / d \ln T=T^{0.5 \pm 0.5}$ for $4.7 \leqslant \log T \leqslant 6.3$.

The most difficult attribute to measure for the $O$ VI-bearing gas is the average volume filling factor $f \equiv\left\langle n_{\varepsilon}\right\rangle^{2} /\left\langle n_{e}^{2}\right\rangle$. Contrary to what is seen for the low

(4) Paresce, et al. (1983) interpretated the diffuse ultraviolet emission observed by Feldman, et al. (1981) to come from gas within a very narrow range of temperatures: $1.6-2.0 \times 10^{5} \mathrm{~K}$. Strictly speaking, their conclusions would not permit the existence of any power law distribution over a broad temperature range. However it is quite possible that some of the spectral features have been misidentified; the resolution of the apparatus which observed the emission was only $60 \AA$, and the feature identified as N IV] $\lambda 1488$ might be something else, such as $\mathrm{H}_{2}$ fluorescence (Duley and Williams 1980); see, e.g. the emission spectrum shown by Brown et al. (1981) which comes from the Lyman bands of vibrationally excited interstellar $\mathrm{H}_{2}$ exposed to L-a emission. If the $\mathrm{N}$ IV identification is indeed incorrect, a power law in temperature would be permitted (and the inferred emission measure could be lower). 
energy x-ray background (McCammon, et al. 1983), there seems to be no anticorrelation between the excesses or deficiencies of $N(O$ VI) per unit distance and variations of normal (cool) interstellar material, as evidenced by the reddenings to the respective stars. From an upper limit to this anticorrelation, Jenkins $(1978 \mathrm{~b})$ concluded that $f<0.2$, provided most of interstellar space is filled with the gas responsible for the reddening and not some other phase at low density. If $f$ were considerably less than this upper limit, we would conclude from the overall average for $n(0 \mathrm{VI})$ that the pressure of the coronal gas would exceed a reasonable upper bound for the pressure of most of the interstellar medium, $p / k=10^{4} \mathrm{~cm}^{-3} \mathrm{~K}$.

\section{c) Comparison with Diffuse Background Emissions}

In simplest terms, a measure of $N(\mathrm{O} \mathrm{VI})$ can be translated into an integral of the electron density $n_{e}$ over a well defined path for gases at temperatures near the peak of the curve for the $O V I$ ion fraction. The intensities of the soft $x$-ray $[130-850 \mathrm{eV}]$ and EUV [50 - $100 \mathrm{eV}]$ emission backgrounds, which arise chiefly from collisional excitations and radiative decay of the electronic levels of highly ionized atoms, allow us to determine the integral of $n_{\theta}^{2}$ for the high-temperature phases over paths of somewhat indefinite length, in large part defined by absorption phenomena from intervening or interspersed denser material ("normal" interstellar matter). Thus, the observations of absorption and emission by these atoms are complementary, since they sample the hot material in different ways. We must acknowledge, of course, that the conclusions will have some errors attributable to not only the observations themselves, but also to uncertainties in temperatures of the gas, the relative abundances of key elements, and the calculations of ion fractions (and, for the x-ray and EUV results, the excitation cross sections). Indeed, the ion fractions are probably not governed by an equilibrium, since the time scale for heating or cooling of the gas could be substantially shorter than the equilibration time for collisional ionization and recombinations.

It is generally believed that the immediate neighborhood of the Sun contains a gaseous medium which is partially ionized with $n(\mathrm{H}) \sim 0.1 \mathrm{~cm}^{-3}$ and $T \sim 1-2 \times 10^{4} \mathrm{~K}$ (Weller and Meier 1981, Dalaudier, et al. 1983). In turn, this local complex of warm gas is surrounded by a volume roughly $100 \mathrm{pc}$ in diameter which may be almost completely filled with hot gas (Sanders, et al. 1977, Tanaka and Bleeker 1977, Hayakawa, et al. 1978, Kraushaar, 1979, Fried, et al. 1980, Arnaud and Rothenflug 1984). This conclusion follows from the observation that a good portion of the $x$-ray background at lower energies is fairly uniform over the whole sky, and this emission seems not to have undergone any (energy dependent) absorption short of reaching known dense clouds which are approximately 100 pc away. A general picture where neutral hydrogen and hot plasmas are intermingling over small scales seems to be disfavored by the steepness of the spectrum of the EUV/soft x-ray emission between 50 and $190 \mathrm{eV}$ (Paresce and Stern 1981). The x-ray results indicate that the emission measure of the hot bubble is generally of order $1-3 \times 10^{-9} \mathrm{~cm}^{-6} \mathrm{pc}$ if there is virtually no absorption $\left[N(\mathrm{H})<<10^{20} \mathrm{~cm}^{-2}\right]$ and $\mathrm{T} \sim$ $10^{6} \mathrm{~K}$ (a temperature giving the most emission in the $\mathrm{B}$ and $\mathrm{C} x$-ray bands). ${ }^{(5)}$ on

(5) In certain directions, not necessarily associated with identifable supernova remnants, the emission measure reaches about $10^{-2} \mathrm{~cm}^{-6}$ pc. (McCammon, et al. 1983, Rocchia, et al. 1984) 
the basis of the x-ray data alone, one must conclude that the pressure of the gas $p / k \geq 10^{4} \mathrm{~cm}^{-3} \mathrm{~K}$. While this local pressure seems to exceed by a substantial margin the general value of about $2-3 \times 10^{3} \mathrm{~cm}^{-3} \mathrm{~K}$, it is not out of the question that the Sun is inside a supernova remnant $100 \mathrm{pc}$ in diameter having an internal temperature $\mathrm{T} \sim 10^{6} \mathrm{~K}$, age $\mathrm{t} \sim 1.4 \times 10^{5} \mathrm{yr}$ and a thermal energy $\sim 3 \times 10^{50} \mathrm{erg}$ (Cox and Anderson, 1982).

When we try to reconcile the $O$ VI data with the $x$-ray and EUV emission measures, we find that there is too little $\mathrm{O} V \mathrm{~V}$ for the amount of emission seen, if the gas were distributed fairly uniformly. One way to resolve the problem is to say that the hot gas is very patchy (i.e., $f$ is low) and rather dense within the patches, but then the inferred pressure of the medium rises. A discussion of this idea was originally presented by Shapiro and Field (1976) who derived remarkably high pressures, but more recent, refined interpretations seem to indicate that the apparent deficiency of $\mathrm{OVI}$ is not too severe.

Burstein, et al. (1977) found that the relative strengths of the x-rays in three different energy bands could only be explained by having the emission come from gases at two very different temperatures [also see de Korte, et al. 1976. A dissenting view has been presented by Hayakawa (1979) however.] They estimated that the lower temperature regime $\left(\mathrm{T} \lesssim 10^{6} \mathrm{~K}\right.$ ) was responsible for about half of the emission sensed by their lowest energy [B band: $130-190 \mathrm{eV}$ ] detector; these temperatures are not too far removed from the range where the $O$ VI is produced. For the power-law temperature distribution defined earlier [\$IIb], one can make half of the Wisconsin group's average B-band count rate consistent with $\left\langle n_{\mathrm{b}}\right\rangle$ from the 0 VI data if $p / k=4 \pm 1.5 \times 10^{4} \mathrm{~cm}^{-3} \mathrm{~K}$, if one assumes for the $\mathrm{x}$-rays that there is very little foreground absorption $\left[\mathrm{N}(\mathrm{H})<2 \times 10^{19} \mathrm{~cm}^{-2}\right]$ and $\mathrm{d}=100 \mathrm{pc}{ }^{(6)}$

A comparison of the $O$ VI data with EUV emission intensities is less sensitive to uncertainties in temperatures, since the most EUV emission comes from a plasma at $T$ best suited for $O \mathrm{VI}$ production. Paresce and Stern (1981) found $n(\mathrm{O} \mathrm{VI})$ too low by a factor 5-10 if $p / k$ is set to $10^{4} \mathrm{~cm}^{-3} \mathrm{~K}$, again assuming the emission is virtually unabsorbed by intervening neutral gases and the existence of a power-law distribution for hot gas temperatures. However they noted that the local $n(0 \mathrm{VI})$ could be somewhat higher than the overall average (see last para. of $\S$ Ila above); by stretching their lower limit for the EUV emission downward by $50 \%$ and assuming the local $n(\mathrm{O} \mathrm{VI})=1.7 \times 10^{-7} \mathrm{~cm}^{-3}$, they could arrive at $f=0.4$ and $p / k=1.5 \times 10^{4} \mathrm{~cm}^{-3} \mathrm{~K}$.

\section{d) Interpretation}

A principal theme of the theoretical description of the interstellar medium by McKee and Ostriker (1977) is that a very inhomogeneous mixture of gas phases is subjected to shocks from supernova explosions. These shocks propagate rather freely through the intercloud medium and heat it to temperatures of around $5 \times 10^{5}$

(6) This result is obtained by evaluating $p / k=\frac{(1.91) 25 \text { counts } s^{-1}}{(100 \mathrm{pc}) C_{8} \ln 10 \int_{0.15}^{8.55} T^{\eta-1} R_{B} d \log T}$, where $\eta=$

$0.5 \pm 0.5$ and $C_{e}$ are as defined by Jenkins (1978b) from the $O$ VI survey, and $R_{B}$ is the B-band emissivity vs. temperature shown in fig. 11a of McCammon, et al. (1983). 
K. Since the isothermal sound speed in this gas is about $80 \mathrm{~km} \mathrm{~s}^{-1}$ and there is probably considerable turbulence, one might expect that there may be much more $O \mathrm{VI}$ in the medium than observed, but that this additional $\mathrm{O} V \mathrm{VI}$ has not been seen because its large velocity dispersion makes the profiles difficult to distinguish from the undulating continua in the spectra of the target stars. Thus the ultraviolet absorption measurements may be badly biased toward only those 0 VI components which have a low velocity dispersion, and this may explain why there is an apparent deficiency relative to the soft $x$-ray and EUV backgrounds.

There is some indication that the components which are observed in $\mathrm{OVI}$ come only from interfaces undergoing conduction and evaporation between the cool clouds and the hot intercloud medium, whish could explain why the profiles appear to be narrow. Cowie, et al. (1979) found correlations between the end-point velocities of $O$ VI profiles and those of some N II and Si III components which seem to support this interpretation. It is also interesting to note that the statistical description of Jenkins (1978b) for packets of $O \mathrm{VI}$ gas, each with $N(\mathrm{O}$ VI) $10^{13} \mathrm{~cm}^{-2}$, implies that roughly 6 of these regions will be intercepted by a random line of sight $1 \mathrm{kpc}$ long. Although possibly coincidental (or attributable to observational effects), this figure is remarkably close to the 7 to 8 clouds $\mathrm{kpc}^{-1}$ deduced from surveys of visual interstellar lines and extinction by dust (Spitzer 1968). If the interpretation that we are seeing conductive interfaces is correct, one must employ the theory of such interfaces in concert with models for the clouds and intercloud medium to ascertain the conditions in the medium. It is important to emphasize, however, that we badly need more definitive observations to confirm more directly that the $\mathrm{O}$ VI indeed arises from evaporation zones around clouds.

Finally, one might ask whether we should be embarrassed by the apparent mismatch in pressure between the very local, partially ionized medium with $T \approx 1-2 \times 10^{4} \mathrm{~K}$ and $n \approx 0.1 \mathrm{~cm}^{-3}$, discussed earlier, and the much hotter, x-ray emitting gas which surrounds it. The answer is, "probably not," or at least, "not yet." When our local cloud was suddenly exposed to a big increase in external pressure as it was overtaken by supernova blast wave, an isothermal shock started to progress toward the cloud's interior. If the ratio of external to internal pressures was, say, a factor of 5 , this shock moved at a velocity $5^{\text {/h }}$ times the local sound speed, $v_{s}=10-20 \mathrm{~km} \mathrm{~s}^{-1}$. Over the $1.4 \times 10^{5} \mathrm{yr}$ lifetime for the local supernova remnant calculated by Cox and Anderson (1982), this shock has probably eaten through only the outermost $3-6$ pc of the perimeter of the local cloud. This length is only comparable to, or possibly much less than, the various estimates for the distance to the cloud's edge, which range from 3 or $4 \mathrm{pc}$ (Bruhweiler and Kondo 1982, Bruhweiler 1982), out to about $50 \mathrm{pc}$ in some directions (Paresce 1984).

\section{INTERSTELLLAR SHOCKS}

Except for the Vela Supernova Remnant and one star (15 Mon) (Jenkins, et al. 1976, Jenkins and Meloy 1974), components of $O$ VI at high radial velocity ( $₹ 250$ $\mathrm{km} \mathrm{s}^{-1}$ ) have not been detected. Those which have been seen may be confined within specific regions of enhanced density. The thickness of post-shock, $O$ VI- 
bearing gas in the general intercloud medium could be so large that interactions with small clouds may create enough ricochet shocks and turbulence to wash out the profiles of $\mathrm{O}$ VI, as discussed above. Slower shocks which produce less energetic ionizations may be more coherent and thus more easily observed.

Cohn and York (1977) have observed high velocity features $(50 \lessgtr \mathrm{v} \leqslant 100 \mathrm{~km}$ $\mathrm{s}^{-1}$ ) of C II, C III and Si III, mostly toward stars in active associations (e.g. Orion) or high-latitude stars. The absence of detectable Si II in these components indicates that photoionization is not the primary cause of ionization. Thus, they were not just viewing high speed clumps within ordinary $\mathrm{H}$ II regions. Instead, shocks which are strong enough to create temperatures of $3-8 \times 10^{4} \mathrm{~K}$ are the most likely explanation, according to the calculations of Shull (1977). More elaborate shock models by Shull and McKee (1979), which include the effects of ionizing radiation on the preshock gas, confirm that Si III should be abundant in the immediate post-shock region for a shock travelling at around $100 \mathrm{~km} \mathrm{~s}^{-1}$, but that there should be virtually no Si III farther back where appreciable cooling and recombination have occurred, because of the rapid charge exchange with neutral hydrogen.

Cowie and York (1978a) performed additional Copernicus observations to look for more high velocity features; this survey extension also emphasized low ionization stages of abundant elements which had intrinsically strong lines in the ultraviolet. They synthesized velocity distribution functions based on the statistics of velocity extrema of the strong lines and concluded that shocks which had reached the isothermal phase were extremely rare (Cowie and York 1978b). Combining their upper limits with estimates for supernova energies and birthrates, they concluded that densities for the intercloud regions with large $f$, the primary medium for shock propagation, should either be $>0.1 \mathrm{~cm}^{-3}$ or $<7 \times 10^{-3} \mathrm{~cm}^{-3}$. The high density alternative is based on the containment of high speed isothermal shocks to small enough radii that they are rarely seen, but this possibility seems pretty unlikely in view of the O VI and EUV/X-ray backgrounds and the theory of McKee and Ostriker (1977). The low density possibility is based on the notion that the shocks never have a chance to become isothermal before they escape from the galactic plane (Chevalier and Oegerle 1979, Cox 1981) or run into other supernova cavities (Cox and Smith 1974, Smith 1977). The upper limit given by Cowie and York (1978b) is consistent with McKee and Ostriker's (1977) estimate of $3.5 \times 10^{-3} \mathrm{~cm}^{-3}$ for the typical hot medium.

\section{DIVERGENT CLUES FROM C IV AND SI IV}

The spectrometer on the Copernicus satellite was not well suited for observing the lines of Si $\mathrm{V}$ and $\mathrm{C} \mathrm{N}$ because at these wavelengths it had low sensitivity and uncertain scattered light levels. Hence, in spite of its inferior wavelength resolution, the International Ultraviolet Explorer (IUE) has been more productive in exploring the properties of Si IV and C IV absorptions toward a broad selection of sources. Even though results for the narrow features of these ions toward many stars have appeared in the literature, and suitable spectra for hundreds of other stars exist in the archives, several factors have hampered our progress in achieving a clear interpretation on the lines' origins and significance. First, in many instances strong stellar (and possibly circumstellar) features have made it very 
difficult to achieve a clean differentiation of the narrow, supposedly interstellar components. (Sometimes a fairly drastic overexposure of a spectrum is needed to properly register an interstellar line in trough of a stellar feature: see, for example, fig. 2 of Pettini and West (1982).) Second, and perhaps more important, is that unlike $O$ VI which has a very high ionization potential, we can plausibly attribute the creation of Si IV, C IV, and to some extent even N V to either photoionization from very hot stars (and/or x-ray sources) or, alternatively, to gas which is either part of, similar to, or somehow connected with the hot, coronal phase which is responsible for the $\mathrm{OVI}, \mathrm{EUV}$ and x-ray results discussed in छII. Finally, for gas at low radial velocities, we usually can not use the presence or absence of species at lower ionization to differentiate between these fundamentally different origins because of the inevitable contamination from $\mathrm{H}$ I regions or other $\mathrm{H}$ II regions of lower excitation. (7)

So far, the results of many of the standard tests to discriminate between truly interstellar origins and effects from the target stars (or their immediate neighbors) have not been completely satisfactory; the observations are evidently influenced by a complicated interplay of several phenomena, and more definitive investigations may be needed to unravel the picture. A simple relationship to examine is that of column densities versus distance. Column densities toward stars more distant than $1 \mathrm{kpc}$ should not suffer appreciably from fluctuations due to Poisson statistics, if the sizes and separations of discrete interstellar gas complexes are comparable to what has been seen in other studies of the local part of our galaxy. However both $N(\mathrm{Si} I \mathrm{~V})$ and $N(\mathrm{C}$ IV) vary over almost two orders of magnitude for any narrow interval of distance beyond $1 \mathrm{kpc}$ (Jenkins 1981, Cowie, et al. 1981). A survey of Wolf-Rayet stars by Smith, et al. (1980) showed virtually no correlation of the column densities of these ions with distance. Many upper limits for closer stars obtained from the Copernicus archives by Jenkins (1981) yield column densities for Si IV and C IV which are lower than $10^{13} \mathrm{~cm}^{-2}$, well below a linear extrapolation of the average $N$ per unit distance from the stars at $d>1 \mathrm{kpc}$. Another classic test, comparing the radial velocities of the features with the expected velocities produced by differential galactic rotation at the positions of the stars, was carried out by Cowie, et al. (1981). These results, however, showed too much scatter to indicate whether or not the lines came from the positions of the stars or halfway between.

A very convincing demonstration that some Si IV and C IV arises from the general interstellar medium was presented by Savage and deBoer $(1979,1981)$. In spectra of stars in the Magellanic Clouds, they found features which correspond to velocities in our galaxy, in addition to absorptions from gases attributable to the halo and the Magellanic system. Galactic Si IV features were also seen in the spectrum of 3C273 by York, et al. (1983). How much of these low velocity absorptions apply to highly ionized material within the galactic plane, rather than a transition layer below the halo, is unresolved.

(7) On many occasions, discrete features of $\mathrm{Si} I \mathrm{~V}$ and $\mathrm{C} \mathrm{IV}$ at high velocity have been recorded by IUE for sources within or behind special, violently disturbed regions, such as those near 0 subdwarfs (Bruhweiler and Dean 1983), some binary systems (Bruhweiler, et al. 1880), active OB associations (Phillips and Gondhalekar 1981, Cowie, et al. 1981, Laurent, et al. 1982) or supernova remnants (Jenkins, ot al. 1984). None have been identified with generally distributed material in the local neighborhood, however. 
Attempts to correlate the strengths of Si IV and C IV lines with properties of the target stars have not been very fruitful. Black, et al. (1980) found that the lines did not seem to correlate with the velocities of stellar winds, and Jenkins (1981) found that $N(\mathrm{C} \mathrm{IV}) / N(\mathrm{Si}$ IV) showed no correspondence with the temperatures of the target stars. In support of the existence of collisionally ionized Si IV and $C \Gamma$ in gases at $T \sim 5 \times 10^{4} \mathrm{~K}$, Bruhweiler, et al. $(1979,1980)$ called attention to the fairly restricted range in the ratios of $N(\mathrm{C} I V)$ to $N(\mathrm{Si} I \mathrm{~V})$ in their sample, extending between only 0.8 and 3.7 for most cases. This, they contended, was much less than the spread one would expect for photoionization from the widely divergent mix of stellar temperatures. Nearly all of their stars had $T_{\mathrm{a}}<35,000 \mathrm{~K}$; the calculations of Cowie, et al. (1981) indicate that $N(\mathrm{C} I V)>N(\mathrm{Si} I V)$ in photoionized regions surrounding stars only with $T_{s}>50,000 \mathrm{~K}$. While it is possible that these results indicate that photoionization by stellar photons is not the dominant source of ionization, one could equally well propose that the density of ambient material near these stars is low enough that the lines of sight are almost always influenced by the starlight from nearly all of the stars in an association. This may explain why $N(\mathrm{C}$ IV $) / N(\mathrm{Si}$ IV) does not seem to vary by large amounts and may also account for the hint that observations of different stars within single associations show some coherence (Cowie, et al. 1981).

A conclusive way to demonstrate that collisional ionization is not responsible for the production of Si IV and C IV is to find profiles with velocity dispersions lower than that expected for thermal doppler broadening at temperatures needed to produce these ions [at equilibrium, $T \approx 10^{5} \mathrm{~K}$; see Shull and Van Steenberg (1982).] Slightly lower temperatures could be anticipated if the gas is cooling radiatively, because recombination times are slower than cooling times (Shapiro and Moore 1976)]. Since the resolution of $I U E$ is only $30 \mathrm{~km} \mathrm{~s}^{-1}$, the dispersions must be inferred from the $b$-values derived from doublet ratios. Most of the observations reported in the literature give $b$-values comparable to or greater than the limiting values of 12 and $8 \mathrm{~km} \mathrm{~s}^{-1}$ for the doppler motions of C IV and Si IV, respectively. A noteworthy exception, however, is Dupree and Raymond's (1983) measurement of C IV absorption in the spectrum of Feige 24.

Some, but not all, white dwarfs observed by IUE show prominent features of Si IV, C IV and N V (Bruhweiler and Kondo 1981, Dupree and Raymond 1983, Malina, Basri and Bowyer 1981, Sion and Guinan 1983). The positive measurements to these stars give column densities well above those generally seen within a few hundred pc (Jenkins 1981). However, it is not completely clear whether these enhancements result from the presence of circumstellar material produced by the stars, or alternatively, from the action of these stars on nearby interstellar gas. Calculations by Dupree and Raymond (1983) indicate that hydrogen-rich, hot dwarfs ( $t \approx 6 \times 10^{4} \mathrm{~K}$ ) should be able to ionize enough Si IV and C IV to be seen with IUE if the ambient density is greater than about $0.1 \mathrm{~cm}^{-3}$. (8) If a star has no appreciable helium cutoff at the high energy end of its spectrum, some $\mathrm{N} \mathrm{V}$ and $\mathrm{O}$ VI will also be produced ( $O$ VI can not be seen with IUE however). More data from local white dwarfs may give us a better insight on what proportion of the medium is filled with gas at moderate densities, as opposed to the very low density

(8) No allowance was made for absorption of the ionizing photons by dust, however, so the actual yields may be somewhat lower than those estimated by Dupree and Raymond (1983); see, e.g. Sarazin (1977). 
$\left(n \approx\right.$ few $\times 10^{-3} \mathrm{~cm}^{-3}$ ) hot material. As emphasized by Hills (1972, 1973, 1974), white dwarfs may be a very important source of ionizing radiation for the interstellar medium in the galactic plane. Dupree and Raymond's (1983) calculations combined with further results on the distribution of C IV and Si IV from IUE may give us more insight on this important topic.

\section{FUTURE PROSPECTS}

In years ahead, our ability to investigate the ultravioiet absorption lines will improve dramatically. The High Resolution Spectrograph [HRS] (Brandt, et al. 1982) aboard the Hubble Space Telescope will have a wavelength resolving power of $\lambda / \Delta \lambda=8 \times 10^{4}$, i.e., $\delta \mathrm{v}=4 \mathrm{~km} \mathrm{~s}^{-1}$, in its highest resolution mode (Bottema, $e t$ al. 1984). The tremendous increase in photometric accuracy and resolution over that obtainable with IUE should virtually eliminate the confusion in identifying different parcels of gas at low velocity and allow us to differentiate those which produce the features from highly ionized atoms from those which do not. Information on whether the Si IV and C IV is ionized by uv photons or by collisions in a hot plasma should come from good measurements of the velocity dispersions, provided, of course, that turbulence and/or velocity shears are not too large. Unfortunately, HRS will not be sensitive to wavelengths near the O VI features; to further study $O$ VI we will need a facility such as the Far Ultraviolet Spectroscopic Explorer Columbus, now in the planning stages, which will be designed specifically to do spectroscopy at wavelengths below the efficiency cutoff of $\mathrm{MgF}_{2}$ coated mirrors and conventional uv detector faceplates.

We desperately need absorption line data from ions which have their peak abundances in the range $10^{6} \leqslant T \leqslant 10^{\gamma} \mathrm{K}$ where most of the x-ray emission occurs. One way to observe them is to use a good crystal or transmission grating spectrometer aboard some reasonably large, orbiting $x$-ray facility. York and Cowie (1983) have calculated that the continua of brighter x-ray sources (with intensities $z$ a few $\mathrm{keV} \mathrm{cm}^{-2} \mathrm{~S}^{-1} \mathrm{keV}^{-1}$ ) should give enough signal-to-noise in a reasonable integration time to permit detection of the strongest lines which might arise over a distance of $1 \mathrm{kpc}$. These authors have tabulated an assortment of strong transitions to levels $0.2 \lesssim E \leqslant 1.0 \mathrm{keV}$ above the ground states of appropriate ions.

Another approach for measuring highly ionized species is to record the extremely weak absorption features from some coronal forbidden lines at visible wavelengths, the most promising of which seem to be [Fe X] $\lambda 6375$ and [Fe XIV] 15303. Hobbs (1984) and Hobbs and Albert (1984) have already made a bold attempt to detect these ions in the interstellar medium, but except for some tan talizing results for two stars (reported elsewhere in this volume), they were only able to report rather high upper limits because detector instabilities limited their signal-to-noise ratios to about 350. A very important result from these first attempts, however, was an exploration of where one can expect to find interference from telluric absorption lines, diffuse interstellar bands, and stellar features. These unwanted features, while present, do not seem to offer serious problems. Thus in due course, when the practice of obtaining high dispersion spectra using CCDs becomes more highly refined and we can routinely obtain photon-limited accuracies with tens of millions of counts per velocity bin, there is a chance that 
the realm of absorption features from highly ionized atoms in space, now the private hunting ground of space astronomers, will soon open up to a much wider comrnunity of observers.

The writing of this paper was supported by NASA Grant NAGW-477.

\section{REFERENCES}

Arnaud, M. and Rothenflug, R. 1984, preprint.

Black, J. H. and Dalgarno, A. 1973, Ap. J. (Letters), 184, L101.

Black, J. H., Dupree, A. K., Hartmann, L. W. and Raymond, J. C. 1980, Ap. J., 239, 502.

Blint, R. J., Watson, W. D., and Christensen, R. B. 1976, Ap. J., 205, 634.

Bottema, M., Cushman, G. W., Holmes, A. W., and Ebbets, D. 1984, Proc. SPIE Conf. "Instrumentation in Astronomy $V$ ", in press.

Brandt, J.C. and the HRS Investigation Definition and Experiment Development Teams 1983, in The Space Telescope Observatory, D. N. B. Hall, ed, NASA CP2244, p. 76.

Brown, A., Jordan, C., Millar, T. J., Gondhalekar, P., and Wilson, R. 1981, Nature, $290,34$.

Bruhweiler, F. C. 1982, in Advances in Ultraviolet Astronomy: Four Years of IUE Research, Y. Kondo, J. M. Mead, and R. D. Chapman, eds., NASA Conf. Pub. 2238, p. 125.

Brubweiler, F. C. and Dean, C. A. 1983, Ap. J. (Letters), 274, L87.

Bruhweiler, F. C. and Kondo, Y. 1981, Ap. J. (Letters), 248, L123. 1982, Ap. J., 259, 232.

Bruhweiler, F. C., Kondo, Y., and McCluskey, G. E. 1979, Ap. J. (Letters), 229, L39. 1980, Ap. J., 237, 19.

Burstein, P., Borken, R. J., Kraushaar, W. L., and Sanders, W. T. 1977, Ap. J., 213, 405.

Castor, J., McCray, R., and Weaver, R. 1975, Ap. J. (Letters), 200, L107.

Chevalier, R. A. and Oegerle, W. R. 1979, Ap. J., 227, 398.

Christenseri, R. B., Watson, W. D., and Blint, R. J. 1977, Ap. J., 213, 712.

Cohn, H. and York, D. G. 1977, Ap. J., 216, 408.

Cowie, L. L., Jenkins, E. B., Songaila, A., and York, D. G. 1979, Ap. J., 232, 467.

Cowie, L. L., Taylor, W., and York, D. G. 1981, Apo. J., 248, 528.

Cowie, L. L. and York, D. G. 1978a, Ap. J., 220, 129. $1978 b, A p . J ., 223,876$.

Cox, D. P. 1981, Ap. J., 245, 534.

Cox, D. P. and Anderson, P. R. 1982, Ap. J., 253, 268.

Cox, D. P. and Smith, B. W. 1974, Ap. J. (Letters), 189, L105.

Dalaudier, F., Bertaux, J. L., Kurt, V. G., and Mironova, E. N. 1983, Astr. Ap., in press.

Dalgarno, A. and McCray, R. A. 1972, Ann. Rev. Astr. Ap., 10, 375.

de Korte, P. A. J., Bleeker, J. A. M., Deerenberg, A. J. M., Hayakawa, S., Yamashita, K., and Tanaka, Y. 1976, Astr. Ap., 48, 235.

Duley, W. W. and Williams, D. A. 1980, Ap. J. (Letters), 242, L179.

Dupree, A. K. and Raymond, J. C. 1983, Ap. J. (Letters), 275, L71.

Feldman, P. D., Brune, W. H., and Henry, R. C. 1981, Ap. J. (Letters), 249, L51. 
Field, G. B., Goldsmith, D. W., and Habing, H. J. 1969, Ap. J. (Letters), 155, L149.

Fried, P. M., Nousek, J. A., Sanders, W. T., and Kraushaar, W. L. 1980, Ap. J., 242, 987.

Goldsmith, D. W., Habing, H. J., and Field, G. B. 1969, Ap. J., 158, 173.

Hartmann, J. 1904, Ap. J., 19, 268.

Hayakawa, S. 1979, in X-Ray Astronomy (COSPAR Symposium), W. A. Baity and L. E. Peterson, eds. (Oxford: Pergammon), p.323.

Hayakawa, S., Kato, T., Nagase, F., Yamashita, K., and Tanaka, Y. 1978, Astr. Ap., 62, 21.

Hills, J. G. 1972, Astr. Ap., 17, 155.

1973, Astr. Ap., 26, 197.

1974, Ap. J., 190, 109.

Hobbs, L. M. 1984, Ap. J., 280, 132.

Hobbs, L. M. and Albert, C. E. 1984, Ap. J., in press.

Jenkins, E. B. $1978 \mathrm{a}, A p$. J., 219, 845.

$1978 \mathrm{~b}, A p . J ., 220,107$.

1978c, Comments Astr., 7, 121.

1981, in The Universe at Ultraviolet Wavelengths, R. D. Chapman, ed., NASA Conf. Pub. 2171, p. 541.

Jenkins, E. B. and Meloy D. A. 1974, Ap. J. (Letters), 193, L121.

Jenkins, E. B., Silk, J., and Wallerstein, G. 1976, Ap. J. (Suppl)., 32, 681.

Jenkins, E. B., Wallerstein, G., and Silk, J. 1984, Ap. J., 278, 649.

Jura, M. 1974, Ap. J., 191, 375.

Kraushaar, W. L. 1979, in X-Ray Astronomy (COSPAR Symposium), W. A. Baity and L. E. Peterson, eds. (Oxford: Pergammon), p. 293.

Laurent, C., Paul, J. A., and Pettini, M. 1982, Ap. J., 260, 163.

Malina, R. F., Basri, G., and Bowyer, S. 1981, Bull. AAS, 13, 873.

McCammon, D., Burrows, D. N., Sanders, W. T., and Kraushaar, W. L. 1983, Ap. J., 269, 107.

McKee, C. F. and Ostriker, J. P. 1977, Ap. J., 218, 148.

O'Donnell, E. J. and Watson, W. D. 1974, Ap. J., 191, 89.

Paresce, F, 1984, $A J$, in press.

Paresce, F., Monsignori Fossi, B. C., and Landini, M. 1983, Ap. J. (Letters), 266, L107.

Paresce, F. and Stern, R. 1981, Ap. J., 247, 89.

Pettini, M. and West, K. A. 1982, Ap. J., 260, 561 .

Phillips, A. P. and Gondhalekar, P. M. 1981, MNRAS, 196, 533.

Pikel'ner, S. B. 1967, Astr. Zh. 44, 915 (English trans.: Soviet Astron. A J, 11, 737 [1968]).

Rocchia, R., Arnaud, M., Blondel, C., Cheron, C., Christy, J. C., Rothenflug, R., Schnopper, H. W., and Delvaille, J. P. 1984, Astr. Ap., 130, 53.

Rogerson, J. B., York, D. G., Drake, J. F., Jenkins, E. B., Morton, D. C., and Spitzer, L. 1973, Ap. J. (Letters), 181, L110.

Sanders, W. T., Kraushaar, W. L., Nousek, J. A., and Fried, P. M. 1977, Ap. J. (Letters), 217, L87.

Sarazin, C. L. 1977 Ap. J., 211, 772.

Savage, B. D. and deBoer, K. S. 1979, Ap. J. (Letters), 230, L77. 1981, Ap. J., 243, 460.

Shapiro, P. R. and Field, G. B. 1976, Ap. J., 205, 762.

Shapiro, P. R. and Moore, R. T. 1976, Ap. J., 207, 460. 
Shull, J. M. 1977, Ap. J., 216, 414.

Shull, J. M. and McKee, C. F. 1979, Ap. J., 227, 131.

Shull, J. M. and Van Steenberg, M. 1982, Ap. J. (Suppl.), 48, 95.

Silk, J. 1970, Ap. Letters, 5, 283.

Silk, J. and Brown, R. L. 1971, Ap. J., 163, 495.

Silk, J. and Werner M. W. 1969, Ap. J., 158, 185.

Sion, E. M. and Guinan, E. F. 1983, Ap. J. (Letters), 265, L87.

Slipher, V. M. 1909, Lowell Obs. Bull., no. 51.

Smith, B. W. 1977, Ap. J., 211, 404.

Smith, L. J., Willis, A. J., and Wilson, R. 1980, MNRAS, 191, 339.

Spitzer, L. 1956, Ap. J., 124, 20. 1968, Diffuse Matter in Space, (New York: Wiley Interscience).

Spitzer, L. and Scott, E. H., 1969, Ap. J., 158, 161.

Spitzer, L. and Tomasko, M. G., 1968, Ap. J., 152, 971.

Steigman, G. 1975, $A p . J ., 199,642$.

Tanaka, Y. and Bleeker, J. A. M. 1977, Space Sci. Rev., 20, 815.

Watson, W. D., and Christensen, R. B. 1979, Ap. J., 231, 627.

Weaver, R., McCray, R., Castor, J., Shapiro, P., and Moore, R. 1977, Ap. J., 218, 377.

Weisheit, J. C. 1973, Ap. J., 185, 877.

Weller, C. S. and Meier, R. R. 1981, Ap. J., 246, 386.

Williamson, F. O., Sanders, W. T., Kraushaar, W. L., McCammon, D., Borken, R., and Bunner, A. N. 1974, Ap. J. (Letters), 193, L133.

York, D. G. 1974, Ap. J. (Letters), 193, L127. 1977, Ap. J., 213, 43.

York, D. G. and Cowie, L. L. 1983, Ap. J., 264, 49.

York, D. G., Wu, C. C., Ratcliff, S., Blades, J. C., Cowie, L. L., and Morton, D. C. 1983, Ap. J., 274, 136. 